\title{
BMJ Open Factors of non-responsive or lost-to- follow-up Japanese mothers during the first year post partum following the Japan Environment and Children's Study: a longitudinal cohort study
}

\author{
Mika Kigawa (D) , ${ }^{1}$ Akiko Tsuchida, ${ }^{2,3}$ Kenta Matsumura, ${ }^{3}$ Ayako Takamori, ${ }^{4}$ \\ Mika Ito, ${ }^{5}$ Tomomi Tanaka, ${ }^{3,6}$ Kei Hamazaki, ${ }^{2,3}$ Yuichi Adachi, ${ }^{6}$ Shigeru Saito, ${ }^{5}$ \\ Hideki Origasa, ${ }^{7}$ Hidekuni Inadera, ${ }^{2,3}$ The Japan Environment and Children's Study \\ (JECS) Group
}

To cite: Kigawa M, Tsuchida A, Matsumura $\mathrm{K}$, et al. Factors of non-responsive or lostto-follow-up Japanese mothers during the first year post partum following the Japan Environment and Children's Study: a longitudinal cohort study. BMJ Open 2019;9:e031222. doi:10.1136/ bmjopen-2019-031222

- Prepublication history for this paper is available online To view these files, please visit the journal online (http://dx.doi. org/10.1136/bmjopen-2019031222).

Received 25 April 2019 Revised 12 August 2019 Accepted 24 September 2019

Check for updates

(c) Author(s) (or their employer(s)) 2019. Re-use permitted under CC BY-NC. No commercial re-use. See rights and permissions. Published by BMJ.

For numbered affiliations see end of article.

Correspondence to

Dr Mika Kigawa;

kigawa-c5s@kuhs.ac.jp

\section{ABSTRACT}

Objectives We examined the factors related to lost-tofollow-up of a birth cohort study during the first year after delivery.

Design Longitudinal cohort study.

Setting Questionnaires were provided by mail. Mothers answered the questionnaires about the children twice: at 6 months and 1 year.

Participants of 103062 pregnancies who consented to participate in the Japan Environment and Children's Study (JECS), 93417 mothers were included in the study after excluding those with multiple births, miscarriages or stillbirths and those who withdrew from the study within 1 year after providing informed consent.

\section{Primary and secondary outcome}

measures Participants' socioeconomic status, medical history, health status, health-related behaviours, their children's health conditions and living situations were collected by self-administered questionnaires during pregnancy or 1 month after delivery as the baseline survey. In addition, two self-administered questionnaires were distributed 6 months and 1 year after delivery. Using the response status of the two questionnaires after delivery, participants' follow-up status was divided into four groups. The related factors were examined using logistic regression analysis.

Results Factors positively correlated with lost-to-followup to the questionnaires were postpartum physical conditions, psychological distress during pregnancy, the child's health status at birth, the child's primary caregiver and the number of siblings of the child. Partners' active participation in JECS was associated with a lower lost-tofollow-up rate to the two questionnaires, whereas inactive participation was positively associated with a higher lostto-follow-up rate.

Conclusion The response rate to the questionnaires seems to be related to the interest and understanding of participants' partners. In addition, the response rates are related to participants' physical conditions and living conditions. To decrease lost-to-follow-up rates in consecutive questionnaire surveys within a cohort
Strengths and limitations of this study

- The Japan Environment and Children's Study (JECS) is a nationwide birth cohort study that includes 103062 pregnancies with confirmed obstetric outcomes in the recruitment; $45 \%$ of live births within the study area was covered by JECS.

- The data on the characteristics of the mothers and children who participated in JECS showed similarity with those from Japanese Vital Statistics.

- The sample size of this study was sufficient to examine the risk factors of lost-to-follow-up.

- The study is strengthened by its assessment of the effects of lost-to-follow-up on prevalence estimates as well as the exposure-outcome relationship.

- The study has two limitations: a short follow-up period and inconsistent provision of consent by participants' partners, weakening the effects of the non-participation group.

study, it may be important for investigators to recognise that participants and their motivation in research can be influenced by perceptions they may have regarding the objectives of the research.

\section{INTRODUCTION}

Lost-to-follow-up is one of the problems in longitudinal cohort survey studies. Lost-tofollow-up participants reduce the sample size designated at the study planning stage, negatively affecting statistical power and analysis results. Certain characteristics related to questionnaire response rates may cause a selection bias in the analysis. Previous studies have confirmed that participants' interest in and understanding of the studied disease and health-related behaviours affect questionnaire response rates, particularly in health-related 
studies. Cross-sectional studies have examined the relationship between non-responsive and responsive participants' interest in the study purpose, their socioeconomic status and so on. ${ }^{1-6}$ On the other hand, a study using participants of longitudinal birth cohort study found an association between socioeconomic status and mother's age. ${ }^{7-9}$ However, few studies have examined the factors related to partial follow-up participants. Therefore, it is necessary to examine the characteristics of participants who do not return questionnaires.

The Japan Environment and Children's Study (JECS) is a nationwide birth cohort study that was started in 2011 to examine the environmental factors affecting child health. The study recruited approximately 100000 pregnant women between 2011 and 2014 to collect biosamples, environmental samples and questionnaire information on their children from pregnancy until 13 years of age. ${ }^{10}$ JECS covered approximately $45 \%$ of the total number of live births within the study area in 2013; moreover, the characteristics of the participating mothers and their children were similar to those obtained from Japan's 2013 Vital Statistics Survey. ${ }^{11}$ The follow-up period is currently in progress, and the data on the children's first year have been finalised.

In this study, we examined the factors related to the non-response and/or lost-to-follow-up to the two questionnaires administered to participants by mail during the first year after delivery using data from JECS.

\section{METHODS}

\section{JECS study design}

Participants were recruited by JECS research co-ordinators at JECS collaborating obstetric hospitals when they came for a health check-up during their first trimester. There were two baseline questionnaires that were administered during pregnancy (first trimester and second or third trimester) and 1 month after delivery. These questionnaires were distributed by JECS research co-ordinators at the collaborating obstetric hospitals and were collected by them at the hospitals or by mail. JECS also requested participants' partners (ie, fathers of the children) to participate in the study. For the participating partners, self-administered questionnaires were distributed to them once during the mothers' pregnancy. The partner questionnaires were distributed by JECS research co-ordinators at the collaborating hospitals, or through the participating mothers, and were collected by JECS research co-ordinators through the mothers or by mail. Maternal medical information during pregnancy and at delivery, as well as medical information on the newborn children of the participants, were transcribed by physicians, midwives/nurses and/or JECS research co-ordinators from the medical records of the collaborating hospitals.

One month after delivery, the participating mothers and their children mostly did not use the obstetric hospital for health check-ups; thus, information on JECS participants' children's health and growth metrics was collected through questionnaires. In this study, we examined data on 6-month-old and 1-year-old children. Both questionnaires were distributed and collected by mail.

\section{Study design}

This study used the JECS Group dataset (jecs-an20180131), released in March 2018 for stakeholders. (The dataset supporting the conclusions of this article will be available after the steering committee of the JECS permits its accessibility.)

The number of records in the dataset in the survey is 104065 . The number of pregnancies who participated in this study is 103062 . Of them, 93417 participants were included in the analysis after excluding those with redundant consents due to multiple consents, those with multiple births, abortions, or stillbirths, and those who withdrew from the study within 1 year after informed consent. Participants were classified into four groups using the response status of the two self-administered questionnaires distributed 1 year after delivery (figure 1 ).

The study analysed the following information, collected from self-administered questionnaires at baseline (two kinds), during pregnancy, and 1 month after delivery, and medical records at delivery: education status, annual household income, participants' medical history, delivery conditions (participation in 'satogaeri bunben', ie, returning to the mother's parents' home for delivery), postpartum smoking and alcohol consumption, postpartum physical conditions of the child, the primary caregiver of the child and the number of siblings living with the child. Psychological distress during pregnancy and depression after delivery were evaluated using the Kessler Psychological Distress Scale (K6), Japanese version ${ }^{12}$ and the Edinburgh Postnatal Depression Scale (EPDS), ${ }^{13}$ respectively. Information on the health status of the child at birth (Apgar score, presence or absence of physical anomalies) was collected from medical records at delivery.

Participants' age at delivery was categorised as follows: $<25,25-29,30-34$ and $\geq 35$.

Partner participation was categorised as follows: did not participate, inactive (or delayed refusals) participation (ie, the partner consented to participate in the study but did not return the questionnaire) and active participation (ie, the partner consented to participate in the research and returned the questionnaire).

\section{Definitions}

Participants were divided into the following four groups based on the response status to the two questionnaires administered within 1 year: the 'lost-to-follow-up group' (non-response to both questionnaires), the 'resumed group' (non-response to the questionnaire for 6-month olds only), the 'discontinuation group' (non-response to the questionnaire for 1-year olds only) and the 'continuation group' (responses to both questionnaires).

Regarding the presence or absence of physical anomalies of children, those diagnosed as 'having physical 
Children in the data set jecs-an-20180131 $(n=104,065)$

Number of mothers: 103,062

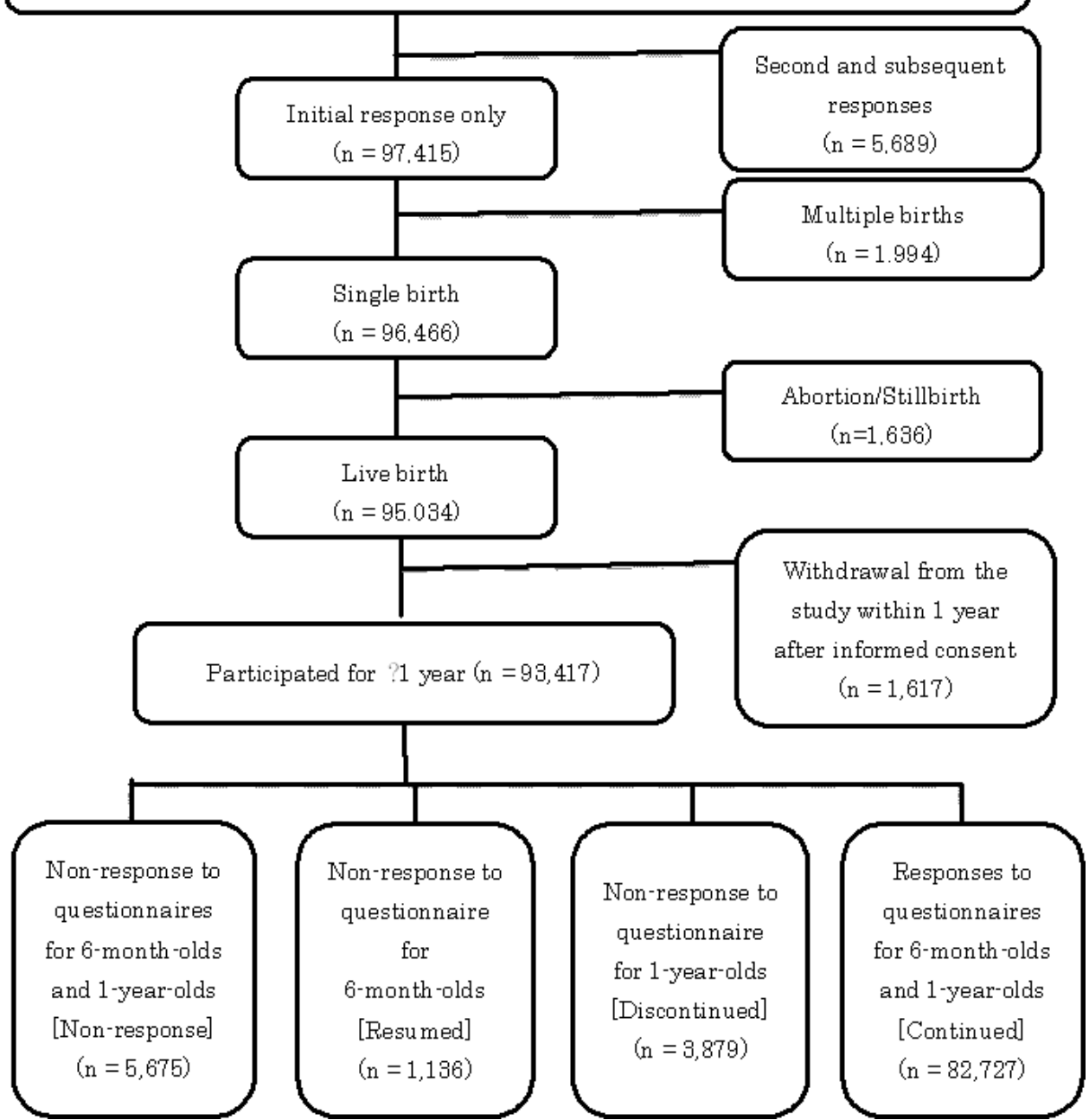

Figure 1 Flow chart of the selection criteria for the four groups based on responses to the two consecutive questionnaires.

anomalies' or 'suspected of having physical anomalies' by an obstetrician were classified as 'having physical anomalies'. ${ }^{14}$ The Apgar score cut-off point was 6/7.

Psychological distress during pregnancy, as measured by the $\mathrm{K} 6$ scale (Japanese version), was defined as $\mathrm{K} 6 \geq 13$, and postpartum depression, as measured by EPDS, was EPDS $\geq 9$.

Postpartum health status of the participants at 1 month after delivery was categorised into the following four categories based on each participant's subjective health status: 'poor', 'fair', 'good', and 'very good.'

Primary caregivers of JECS participants' 1-year-old children were categorised into two groups: 'mother' and 'other than mother'.

The number of siblings living with the child was categorised into three groups: 0,1 and $\geq 2$.

Education status was categorised into two groups: $<13$ years and $\geq 13$ years.

Annual household income was categorised into three groups: $<4$ million yen, $\geq 4$ million and $<12$ million yen, and $\geq 12$ million yen.

\section{Patient and public involvement}

JECS started recruiting expectant mothers in January 2011 with the aim of assessing environmental factors that affect children's health, with the goal of providing a foundation for policymaking to safeguard the environment for the next generation. JECS study aimed to recruit approximately 100000 pregnant women and their partners over 3 years, to collect biological samples, and to collect data on their children until they turned 13 years old. ${ }^{10}$

Written informed consent for participation in JECS was obtained from individual mothers. In addition to the JECS main study, adjunct studies were conducted by the member of JECS Group or any combination of them. The adjunct studies may have included procedures that were not adopted by the main study. This study was one of the adjunct studies of JECS, based on an existing dataset, and hence, patients were not directly involved in the sampling process. 


\section{Analysis methods}

Information on the participants (ie, duration of education, household income, medical history, smoking, alcohol consumption and depression), the health status of the child (ie, Apgar score, presence or absence of physical anomalies), partner participation in JECS and the presence or absence of partner responses to the questionnaires were examined by Pearson's $\chi^{2}$ test or Fisher's exact test. A significance level of 0.01 (two-tailed) was used for all statistical analyses. A binomial logistic regression analysis was performed using significant variables to calculate OR and 95\% CI. JMP Pro V.13 (SAS Institute) was used for all statistical analyses.

\section{RESULTS}

\section{Participant characteristics}

Response rates to the questionnaires (number of returned questionnaires/number of participants) for 6-month olds and 1-year olds were $92.7 \%(86606 / 93417)$ and $89.7 \%$ (83863/93417), respectively.

Participant characteristics are shown in table 1 . The age at delivery was significantly younger in the lost-tofollow-up group than in the other groups. Postpartum prevalence of smoking and alcohol consumption was also significantly higher in the lost-to-follow-up group than in the other groups (although alcohol consumption was the highest in the resumed group). Regarding self-reported health status, in the lost-to-follow-up group, the rate of 'very good' was the lowest among all groups and that of 'poor' was significantly higher compared with the discontinuation and continuation groups. Regarding socioeconomic status, duration of education was significantly shorter in the lost-to-follow-up group than in the other groups. Further, the rate of low-income earners was significantly higher in the lost-to-follow-up group than in the discontinuation and continuation groups, but the rate of high-income earners was also higher in the lostto-follow-up group. Medical history of bronchial asthma also showed a significant negative association with the response rate to the questionnaires. Incidence of psychological distress and/or depression during pregnancy and after delivery was significantly higher in the lost-tofollow-up group.

Regarding problems at delivery, the rate of neonates with Apgar scores less than 7 at 1 min, physical anomalies or who were hospitalised was significantly higher in the lost-to-follow-up group.

In addition, the rate of women who participated in 'satogaeri bunben' at delivery was significantly lower in the lost-to-follow-up group. The rate of mothers who were the primary caregivers of the child was significantly lower in the lost-to-follow-up group. The number of siblings living with the child was significantly higher in the lost-tofollow-up group.

The rate of non-participating partners was significantly higher in the lost-to-follow-up group.

\section{Factors related to lost-to-follow-up}

Factors related to lost-to-follow-up for the discontinuation group, the resumed group and the continuation groups were examined in this study. Table 2 shows the results of a logistic regression analysis of factors related to the lostto-follow-up group.

The higher the mother's age, the greater the likelihood of belonging to the lost-to-follow-up group compared with the resumed group ( $\geq 35$ : OR $1.27,95 \%$ CI: 0.97 to 1.66 ), and the smaller the likelihood of belonging to the lost-tofollow-up group compared with the discontinuation and continuation groups ( $\geq 35$ : OR $0.83,0.40$, prospectively). Smoking habits increased in the lost-to-follow-up group compared with the other groups for current smokers (resumed: OR 1.23; discontinuation: 1.52; continuation group: 3.24). Regarding alcohol consumption, the rate in the lost-to-follow-up group was lower than that in the resumed group (OR 0.87), but higher compared with the discontinuation and continuation groups (OR 1.19, 1.33, respectively). Education status decreased in the lost-tofollow-up group (at $\geq 13$ years) compared with the other groups. Regarding family income, high-income earners increased in the lost-to-follow-up group compared with the other groups; however, the rate of medium-income earners increased in the lost-to-follow-up group compared with the resumed group (OR 1.12), while it decreased compared with the discontinuation and continuation groups (OR 0.92, 0.91, respectively).

The lost-to-follow-up group showed a decrease that depended on the self-reported health status after delivery compared with the other groups, but the OR was 1 or more in the resumed group and less than 1 in the discontinuation and continuation groups. Among all groups, the lost-to-follow-up group showed an increase in psychological distress during pregnancy and depression after delivery.

Regarding physical anomalies, the rate in the lost-tofollow-up group was low compared with the resumed group (OR 0.86) and high compared with the discontinuation and continuation groups (OR 1.34, 1.24, respectively).

Regarding the presence or absence of siblings, the higher the number of siblings, the higher the lost-to-follow-up rate (one sibling: OR 1.13, 1.10, 1.47, respectively; $\geq 2$ siblings: OR 1.28, 1.35, 2.10, respectively). The lost-to-follow-up rate was significantly higher when the primary caregiver of the child was an individual other than the mother (OR 1.52 , 1.61, 2.05, respectively). Regarding the relationship between partner participation and questionnaire response rate, OR for inactive participation was higher than 1 in the lost-to-follow-up group compared with the other groups, but that for active participation was higher than 1 compared with the resumed group and lower than 1 compare compared with the other two groups.

\section{DISCUSSION}

In JECS, information on developmental environment and the growth and development of the children is collected 
Table 1 Baseline characteristics of samples

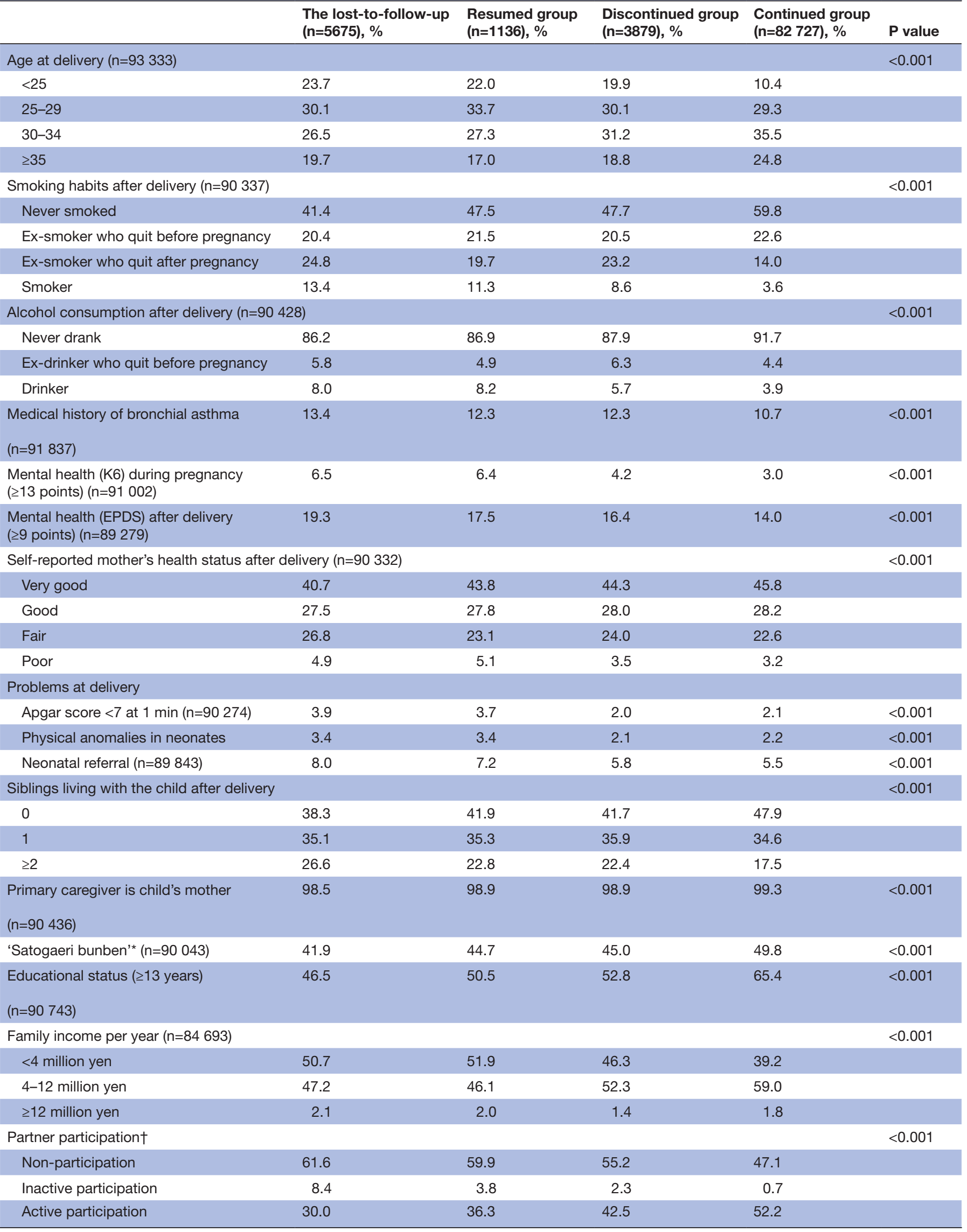


*'Satogaeri bunben' refers to the mother returning to her parents' home for delivery.

†lf the partner agreed to participate but did not return the questionnaire, his/her participation was classified as passive participation. If the partner returned the questionnaire, his/her participation was classified as active participation. Partners who did not agree to participate or could not be contacted to request participation were classified under 'non-participation'.

EPDS, Edinburgh Postnatal Depression Scale; K6, Kessler Psychological Distress Scale.

through the observation of guardians-usually the participating mothers. This study examined factors related to the response rates to the questionnaires for 6-month olds and 1-year olds, using data from approximately 100000 pregnant women participating in JECS. Factors related to follow-up to both questionnaires included postpartum physical conditions, number of siblings, the caregiver of the child at 1 month of age and partner participation. Depression during pregnancy was significantly associated with non-response to the questionnaire for 6-month olds only but not with non-response to the questionnaire for 1-year olds only. There was no significant association between physical anomalies in the child and non-response to both questionnaires. The degree of contribution of each significant factor varied between the two questionnaires. In the resumed group, there were no significant factors contributing to the response rate. In the discontinuation group, having two elder siblings and partial partner participation were significantly associated with higher lost-to-follow-up rates. More factors-such as the health status of the mother, number of siblings of the child, caregiver, partner participation, depression during pregnancy and so on-were related to follow-up rate in the continuation group more than in the other groups.

Many studies have reported that health-related behaviours and the socioeconomic status of questionnaire respondents affect questionnaire response rates. ${ }^{2415-23}$ As with this study, according to some previous studies, low health status at the time the questionnaire is completed is related to non-response in a mail survey. ${ }^{24}{ }^{25}$ While depression during pregnancy is difficult to alleviate, ${ }^{26}$ it has been reported that postpartum depression resolves until 6 months after delivery then relapses. ${ }^{27}$ The difference in the clinical course of depression in pregnant women may affect follow-up rate to the questionnaires for 6-month olds and 1-year olds.

The results of this study are consistent with those of previous studies in showing that health status, number of children and caregiver affect questionnaire response rates. ${ }^{28} 29$

The factor most strongly associated with both questionnaire response rates was partner participation. The results of a survey of people with a history of childhood cancer conducted by Alessi et $a l^{30}$ suggested that patient participation may be affected by whether the general practitioner (GP) working with the patient understands the purpose of the survey. ${ }^{30}$ In this study, we considered the participation status of the partners of participating mothers in JECS. Active participating partners agreed to participate in JECS and returned the questionnaires, so they were considered to be familiar with JECS. They might have played a role similar to that of the GP in Alessi et $a l \mathrm{~s}^{30}$ study; therefore, the lost-to-follow-up rate of participants decreased as a result of having such a partner. On the other hand, regarding inactive participation (delayed refusals), partners who agreed to participate but did not return the questionnaires, this study suggests that having an inactive partner may have increased participants' dropout rate. Therefore, it may be important to make people surrounding the JECS participants understand the purpose of JECS to prevent lost-to-follow-up.

Teague and colleagues examined the effectiveness of methods to prevent dropouts using meta-analysis, but found that methods other than reminders were less effective. ${ }^{31}$ Results of this study show that including participants' surroundings during interventions might prevent dropout.

\section{LIMITATIONS OF THIS STUDY}

This study has the following limitations: (a) the follow-up period was only 1 year and (b) not all partners of the JECS participants were contacted for participation in this study. As the dropout rate only during the first year of follow-up was examined, factors affecting the lost-to-follow-up rate for longer periods are not clear. The partners of the participants were contacted in person by the investigator during the pregnancy of the participants to obtain informed consent. However, in cases in which the investigators were not able to contact the partner to request participation, it was classified as participation refusal in the dataset because of the lack of informed consent. This study's strengths lie in its sample size and the participants' characteristics. There were 93417 participants, which is sufficient for examining the risk factors of the lost-tofollow-up group. JECS covered about $45 \%$ of live births within the study area, and the characteristics of mothers who participated in JECS were similar to those of mothers in the Japanese Vital Statistics. Therefore, the results of this study have sufficient power for our analysis.

\section{Author affiliations}

${ }^{1}$ Department of Liberal Arts and Human Development, Kanagawa University of Human Services, Yokosuka, Japan

${ }^{2}$ Department of Public Health, Faculty of Medicine, University of Toyama, Graduate School of Medicine and Pharmaceutical Sciences, Toyama, Japan 
Table 2 Multivariate logistic regression predicting the likelihood of survey non-response of each questionnaire

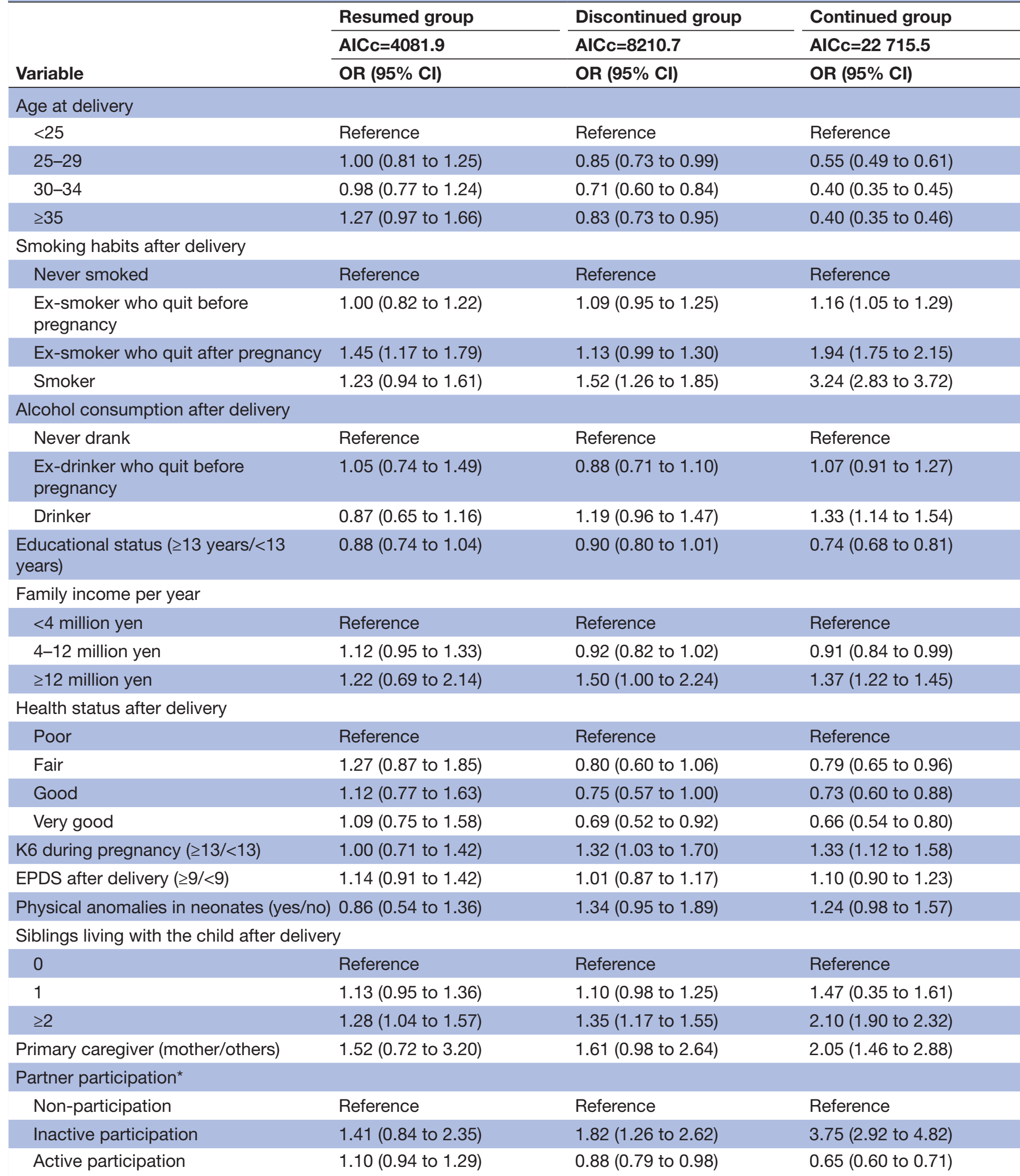

*If the partner agreed to participate but did not return the questionnaire, his participation was classified as inactive participation. If the partner returned the questionnaire, his participation was classified as active participation. Partners who did not agree to participate or could not be contacted to request participation were classified under 'non-participation'.

AIC, Akaike's information criterion; EPDS, Edinburgh Postnatal Depression Scale; K6, Kessler Psychological Distress Scale. 
${ }^{3}$ Toyama Regional Centre for Japan Environment and Children's Study, University of Toyama, Toyama, Japan

${ }^{4}$ Clinical Research Centre, Saga University Hospital, Saga, Japan

${ }^{5}$ Department of Obstetrics and Gynecology, Faculty of Medicine, University of Toyama, Graduate School of Medicine and Pharmaceutical Science for Education, Toyama, Japan

${ }^{6}$ Department of Pediatrics, University of Toyama, Faculty of Medicine, Graduate School of Medicine and Pharmaceutical Science for Education, Toyama, Japan ${ }^{7}$ Division of Biostatistics and Clinical Epidemiology, Faculty of Medicine, University of Toyama, Graduate School of Medicine and Pharmaceutical Sciences, Toyama, Japan

Acknowledgements We appreciate the children and their families who participated in and co-operated with this study. We also thank all members of JECS as of 2019: Toshihiro Kawamoto (Principal Investigator, National Institute for Environmental Studies, Tsukuba, Japan), Yukihiro Ohya (National Center for Child Health and Development, Tokyo, Japan), Reiko Kishi (Hokkaido University, Sapporo, Japan), Nobuo Yaegashi (Tohoku University, Sendai, Japan), Koichi Hashimoto (Fukushima Medical University, Fukushima, Japan), Chisato Mori (Chiba University, Chiba, Japan), Shuich Ito (Yokohama City University, Yokohama, Japan), Zentaro Yamagata (University of Yamanashi, Chuo, Japan), Hidekuni Inadera (University of Toyama, Toyama, Japan), Michihiro Kamijima (Nagoya City University, Nagoya, Japan), Takeo Nakayama (Kyoto University, Kyoto, Japan), Hiroyasu Iso (Osaka University, Suita, Japan), Masayuki Shima (Hyogo College of Medicine, Nishinomiya, Japan), Yasuaki Hirooka (Tottori University, Yonago, Japan), Narufumi Suganuma (Kochi University, Nankoku, Japan), Koichi Kusuhara (University of Occupational and Environmental Health, Kitakyushu, Japan) and Takahiko Katoh (Kumamoto University, Kumamoto, Japan).

Collaborators Toshihiro Kawamoto, Yukihiro Ohya, Reiko Kishi, Nobuo Yaegashi, Koichi Hashimoto, Chisato Mori, Shuich Ito, Zentaro Yamagata, Michihiro Kamijima, Takeo Nakayama, Hiroyasu Iso, Masayuki Shima, Yasuaki Hirooka, Narufumi Suganuma, Koichi Kusuhara, Takahiko Katoh.

Contributors MK designed and carried out this study, performed the statistical analysis and wrote the manuscript. ATsuchida and ATakamori carried out data collection and helped in drafting the manuscript. KM assisted with statistical analysis. Ml and TT carried out data collection and helped in critically revising the manuscript. $\mathrm{KH}$ and $\mathrm{HI}$ participated in the design of the study and helped in critically revising the manuscript. $\mathrm{HO}$ assisted with statistical analysis. YA and SS helped in critically revising the manuscript. The JECS Group assisted in data collection. All authors have read and approved the final manuscript.

Funding The Japan Environment and Children's Study was funded by the Ministry of the Environment, Japan. The findings and conclusions of this article are solely the responsibility of the authors and do not represent the official views of the abovementioned government.

Competing interests None declared.

Patient consent for publication Not required.

Ethics approval The JECS protocol was reviewed and approved by the Ministry of the Environment's Institutional Review Board on Epidemiological Studies and by the Ethics Committees of all participating institutions. Informed consent was obtained from all participants in written form.

Provenance and peer review Not commissioned; externally peer reviewed.

Data availability statement Data are available upon reasonable request.

Open access This is an open access article distributed in accordance with the Creative Commons Attribution Non Commercial (CC BY-NC 4.0) license, which permits others to distribute, remix, adapt, build upon this work non-commercially, and license their derivative works on different terms, provided the original work is properly cited, appropriate credit is given, any changes made indicated, and the use is non-commercial. See: http://creativecommons.org/licenses/by-nc/4.0/.

\section{ORCID iD}

Mika Kigawa http://orcid.org/0000-0002-8131-5682

\section{REFERENCES}

1 Austin MA, Criqui MH, Barrett-Connor E, et al. The effect of response bias on the odds ratio. Am J Epidemiol 1981;114:137-43.
2 Christensen Al, Ekholm O, Gray L, et al. What is wrong with nonrespondents? Alcohol-, drug- and smoking-related mortality and morbidity in a 12-year follow-up study of respondents and nonrespondents in the Danish health and morbidity survey. Addiction 2015;110:1505-12.

3 Korkeila K, Suominen S, Ahvenainen J, et al. Non-response and related factors in a nation-wide health survey. Eur J Epidemiol 2001;17:991-9.

4 Pietilä A-M, Rantakallio P, Läärä E. Background factors predicting non-response in a health survey of northern Finnish young men. Scand J Soc Med 1995;23:129-36.

5 Schneider KL, Clark MA, Rakowski W, et al. Evaluating the impact of non-response bias in the behavioral risk factor surveillance system (BRFSS). J Epidemiol Community Health 2012;66:290-5.

6 Stang A. Nonresponse research--an underdeveloped field in epidemiology. Eur J Epidemiol 2003;18:929-32.

7 Young AF, Powers JR, Bell SL. Attrition in longitudinal studies: who do you lose? Aust N Z J Public Health 2006;30:353-61.

8 Marete I, Tenge C, Chemweno C, et al. Lost to follow-up among pregnant women in a multi-site community based maternal and newborn health registry: a prospective study. Reprod Health 2015;12 Suppl 2:s4.

9 Cameron CM, Osborne JM, Spinks AB, et al. Impact of participant attrition on child injury outcome estimates: a longitudinal birth cohort study in Australia. BMJ Open 2017;7:e015584.

10 Kawamoto T, Nitta H, Murata K, et al. Rationale and study design of the Japan environment and children's study (JECS). BMC Public Health 2014:14:25.

11 Michikawa T, Nitta H, Nakayama SF, et al. Baseline profile of participants in the Japan environment and children's study (JECS). $J$ Epidemiol 2018;28:99-104.

12 Furukawa TA, Kawakami N, Saitoh M, et al. The performance of the Japanese version of the K6 and K10 in the world mental health survey Japan. Int J Methods Psychiatr Res 2008;17:152-8.

13 Okano T, Murata M, Masuji F, et al. Validation and reliability of Japanese version of EPDS (Edinburgh postnatal depression scale. Archives of psychiatric diagnostics and clinical evaluation 1996;7:525-33.

14 Mezawa H, Tomotaki A, Yamamoto-Hanada K, et al. Prevalence of congenital anomalies in the Japan environment and children's study. J Epidemiol 2019:29:247-56. JE20180014.

15 Abrahamsen R, Svendsen MV, Henneberger PK, et al. Non-response in a cross-sectional study of respiratory health in Norway. BMJ Open 2016:6:e009912.

16 Barchielli A, Balzi D. Nine-Year follow-up of a survey on smoking habits in Florence (Italy): higher mortality among non-responders. Int $J$ Epidemiol 2002;31:1038-42.

17 Jacobsen TN, Nohr EA, Frydenberg M. Selection by socioeconomic factors into the Danish national birth cohort. Eur J Epidemiol 2010;25:349-55.

18 Van Loon AJM, Tijhuis M, Picavet HSJ, et al. Survey non-response in the Netherlands: effects on prevalence estimates and associations. Ann Epidemiol 2003;13:105-10.

19 Macera CA, Jackson KL, Davis DR, et al. Patterns of non-response to a mail survey. J Clin Epidemiol 1990;43:1427-30.

20 Madigan MP, Troisi R, Potischman N, et al. Characteristics of respondents and non-respondents from a case-control study of breast cancer in younger women. Int J Epidemiol 2000;29:793-8.

21 Nakai S, Hashimoto S, Murakami Y, et al. [Response rates and nonresponse bias in a health-related mailed survey]. Nihon Koshu Eisei Zasshi 1997;44:184-91.

22 Nieminen T, Prättälä R, Martelin T, et al. Social capital, health behaviours and health: a population-based associational study. BMC Public Health 2013;13:613.

23 Tolonen H, Laatikainen T, Helakorpi S, et al. Marital status, educational level and household income explain part of the excess mortality of survey non-respondents. Eur J Epidemiol 2010;25:69-76.

24 Dengler R, Roberts H, Rushton L. Lifestyle surveys--the complete answer? J Epidemiol Community Health 1997;51:46-51.

25 Etter JF, Perneger TV. Analysis of non-response bias in a mailed health survey. J Clin Epidemiol 1997;50:1123-8.

26 Sexton MB, Flynn HA, Lancaster C, et al. Predictors of recovery from prenatal depressive symptoms from pregnancy through postpartum. $J$ Womens Health 2012;21:43-9.

27 Dagher RK, McGovern PM, Dowd BE. Maternity leave duration and postpartum mental and physical health: implications for leave policies. J Health Polit Policy Law 2014;39:369-416.

28 Corry NH, Williams CS, Battaglia M, et al. Assessing and adjusting for non-response in the millennium cohort family study. BMC Med Res Methodol 2017:17:16. 
29 Long AC, Downey L, Engelberg RA, et al. Understanding response rates to surveys about family members' psychological symptoms after patients' critical illness. J Pain Symptom Manage 2017;54:96-104.
30 Alessi D, Pastore G, Zuccolo L, et al. Analysis of nonresponse in the assessment of health-related quality of life of childhood cancer survivors. Eur J Cancer Prev 2007;16:576-80.

31 Teague S, Youssef GJ, Macdonald JA, et al. Retention strategies in longitudinal cohort studies: a systematic review and meta-analysis. BMC Med Res Methodol 2018;18:151. 